Open Access

Ganlin Zhao*

\title{
Crystal structure of bis $\left(\mu_{3}-2,2^{\prime}\right.$-azanediylbis (ethan-1-olato)- $\mathrm{K}^{5} \mathrm{O}: 0, \mathrm{~N}, \mathrm{O}^{\prime}: \mathrm{O}^{\prime}$ )-tetrachlorido- bis $\left(\mu_{2}-2-((2-h y d r o x y e t h y l) a m i n o) e t h a n-1-o l a t o-\right.$ $\left.\mathrm{K}^{3} \mathrm{~N}, \mathrm{O}: \mathrm{O}\right)$ dicobalt(II)dicobalt(III), $\mathrm{C}_{16} \mathrm{H}_{38} \mathrm{Cl}_{4} \mathrm{Co}_{4} \mathrm{~N}_{4} \mathrm{O}_{8}$
}

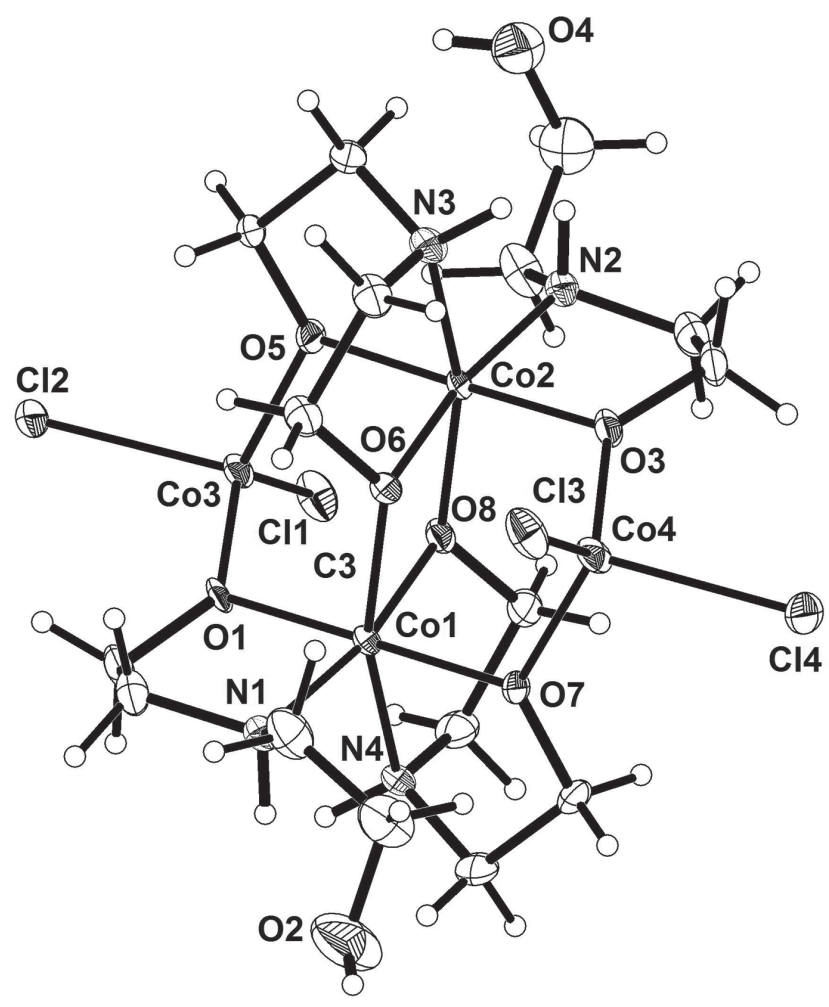

DOI 10.1515/ncrs-2017-0005

Received January 6, 2017; accepted June 16, 2017; available online July 6, 2017

\section{Abstract}

$\mathrm{C}_{16} \mathrm{H}_{38} \mathrm{Cl}_{4} \mathrm{Co}_{4} \mathrm{~N}_{4} \mathrm{O}_{8}$, monoclinic, $P 2_{1} / c \quad$ (no. 14), $\quad a=$

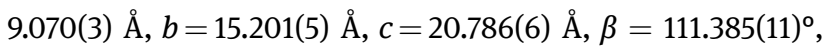
$V=2668.5(15) \AA^{3}, Z=4, R_{\mathrm{gt}}(F)=0.0646, w R_{\mathrm{ref}}\left(F^{2}\right)=0.1743$, $T=293 \mathrm{~K}$.

CCDC no.: 1556624

*Corresponding author: Ganlin Zhao, Changsha Enviromental Protection College, 410004, Changsha, Hunan, People's Republic of China, e-mail: ganlinzhao16@163.com
Table 1: Data collection and handling.

\begin{tabular}{ll}
\hline Crystal: & Green block \\
Size: & $0.20 \times 0.18 \times 0.15 \mathrm{~mm}$ \\
Wavelength: & Mo $K \alpha$ radiation $(0.71073 \AA$ A $)$ \\
$\mu:$ & $28.9 \mathrm{~cm}^{-1}$ \\
Diffractometer, scan mode: & Bruker APEXII, $\varphi$ and $\omega$ \\
$2 \theta_{\text {max }}$, completeness: & $50.2^{\circ},>99 \%$ \\
$N(h k l)_{\text {measured }}, N(h k l)_{\text {unique }}, R_{\text {int }}:$ & $31371,4718,0.065$ \\
Criterion for $I_{\text {obs }}, N(h k l)_{\text {gt }}:$ & $I_{\text {obs }}>2 \sigma\left(I_{\text {obs }}\right), 3958$ \\
$N(\text { param })_{\text {refined }}:$ & 321 \\
Programs: & Bruker [1], SHELX [2] \\
\hline
\end{tabular}

The asymmetric unit of the title crystal structure is shown in the figure. Tables 1 and 2 contain details on crystal structure and measurement conditions and a list of the atoms including atomic coordinates and displacement parameters.

\section{Source of material}

A mixture of $\mathrm{CoCl}_{2} \cdot 6 \mathrm{H}_{2} \mathrm{O} \quad(0.1 \mathrm{mmol})$, diethanolamine $(0.1 \mathrm{mmol})$, triethylamine $(0.1 \mathrm{~mL})$ and water $(15 \mathrm{~mL})$ was stirred for ten minutes at room temperature. The mixture was then transfered in a $20 \mathrm{~mL}$ Teflon-lined stainless-steel reactor, heated to $433 \mathrm{~K}$ for $72 \mathrm{~h}$, and then slowly cooled to room temperature at a rate of $5 \mathrm{~K} / \mathrm{h}$. Dark green block crystals were collected by filtration.

\section{Experimental details}

All $\mathrm{H}$ atoms were placed in idealized positions, refined with distance restraints of $\mathrm{C}-\mathrm{H}=0.97 \AA$ and $\mathrm{N}-\mathrm{H}=0.91 \AA$ And refined as riding atoms with $U_{\text {iso }}(\mathrm{H})=1.2 U_{\text {eq }}(\mathrm{C}, \mathrm{N})$, respectively.

\section{Discussion}

The construction of polynuclear metal complexes is one of the most active research areas in coordination chemistry, not only because of their intriguing variety of structures but also due to their potential applications as functional materials used in the area of molecular magnetism, quantum computing and biology [3-10]. It is well known that the structures of the 
Table 2: Fractional atomic coordinates and isotropic or equivalent isotropic displacement parameters $\left(\AA^{2}\right)$.

\begin{tabular}{|c|c|c|c|c|}
\hline Atom & $x$ & $y$ & $z$ & $U_{\text {iso }} * / U_{\text {eq }}$ \\
\hline C1 & $0.2209(9)$ & $0.0686(5)$ & $0.3228(4)$ & $0.0269(16)$ \\
\hline $\mathrm{H} 1 \mathrm{~A}$ & 0.1265 & 0.0392 & 0.2921 & $0.032^{\star}$ \\
\hline $\mathrm{H} 1 \mathrm{~B}$ & 0.2935 & 0.0243 & 0.3505 & $0.032^{*}$ \\
\hline $\mathrm{C} 2$ & $0.1794(8)$ & $0.1307(5)$ & $0.3678(4)$ & $0.0226(15)$ \\
\hline $\mathrm{H} 2 \mathrm{~A}$ & 0.0880 & 0.1652 & 0.3406 & $0.027^{*}$ \\
\hline $\mathrm{H} 2 \mathrm{~B}$ & 0.1525 & 0.0984 & 0.4023 & $0.027^{\star}$ \\
\hline C3 & $0.3751(9)$ & $0.0587(6)$ & $0.2474(4)$ & $0.0325(18)$ \\
\hline $\mathrm{H} 3 \mathrm{~A}$ & 0.4804 & 0.0454 & 0.2800 & 0.039 * \\
\hline $\mathrm{H} 3 \mathrm{~B}$ & 0.3160 & 0.0040 & 0.2372 & 0.039 * \\
\hline $\mathrm{C} 4$ & $0.3896(10)$ & $0.0911(6)$ & $0.1829(4)$ & $0.039(2)$ \\
\hline $\mathrm{H} 4 \mathrm{~A}$ & 0.4495 & 0.1456 & 0.1917 & $0.047^{\star}$ \\
\hline $\mathrm{H} 4 \mathrm{~B}$ & 0.4452 & 0.0482 & 0.1656 & $0.047^{*}$ \\
\hline C5 & $0.9019(9)$ & $0.3882(5)$ & $0.4864(4)$ & $0.0275(16)$ \\
\hline $\mathrm{H} 5 \mathrm{~A}$ & 0.8233 & 0.4307 & 0.4601 & $0.033^{*}$ \\
\hline $\mathrm{H} 5 \mathrm{~B}$ & 0.9936 & 0.4199 & 0.5169 & $0.033^{*}$ \\
\hline C6 & $0.9479(8)$ & $0.3307(5)$ & $0.4391(4)$ & $0.0239(15)$ \\
\hline $\mathrm{H} 6 \mathrm{~A}$ & 0.9640 & 0.3658 & 0.4032 & 0.029 * \\
\hline H6B & 1.0461 & 0.3004 & 0.4645 & 0.029 * \\
\hline $\mathrm{C} 7$ & $0.7657(9)$ & $0.3812(5)$ & $0.5713(4)$ & $0.0317(18)$ \\
\hline $\mathrm{H} 7 \mathrm{~A}$ & 0.6893 & 0.3444 & 0.5813 & $0.038^{*}$ \\
\hline $\mathrm{H} 7 \mathrm{~B}$ & 0.7104 & 0.4324 & 0.5461 & $0.038^{*}$ \\
\hline $\mathrm{C} 8$ & $0.8945(11)$ & $0.4107(6)$ & $0.6394(4)$ & $0.039(2)$ \\
\hline $\mathrm{H} 8 \mathrm{~A}$ & 0.9696 & 0.4488 & 0.6297 & $0.047^{\star}$ \\
\hline $\mathrm{H} 8 \mathrm{~B}$ & 0.8467 & 0.4435 & 0.6668 & $0.047^{*}$ \\
\hline C9 & $0.8486(8)$ & $0.1319(5)$ & $0.5822(4)$ & $0.0219(15)$ \\
\hline $\mathrm{H} 9 \mathrm{~A}$ & 0.9085 & 0.1773 & 0.6137 & $0.026^{*}$ \\
\hline H9B & 0.8914 & 0.0751 & 0.6013 & $0.026^{*}$ \\
\hline C10 & $0.6743(7)$ & $0.1376(5)$ & $0.5731(3)$ & $0.0193(14)$ \\
\hline $\mathrm{H} 10 \mathrm{~A}$ & 0.6216 & 0.0823 & 0.5558 & $0.023^{*}$ \\
\hline $\mathrm{H} 10 \mathrm{~B}$ & 0.6640 & 0.1502 & 0.6170 & $0.023^{*}$ \\
\hline C11 & $0.8162(8)$ & $0.0642(4)$ & $0.4685(4)$ & $0.0227(15)$ \\
\hline $\mathrm{H} 11 \mathrm{~A}$ & 0.8396 & 0.0119 & 0.4972 & $0.027^{*}$ \\
\hline $\mathrm{H} 11 \mathrm{~B}$ & 0.8785 & 0.0619 & 0.4392 & $0.027^{\star}$ \\
\hline $\mathrm{C} 12$ & $0.6396(8)$ & $0.0658(4)$ & $0.4234(4)$ & $0.0215(14)$ \\
\hline $\mathrm{H} 12 \mathrm{~A}$ & 0.6155 & 0.0218 & 0.3872 & $0.026^{*}$ \\
\hline $\mathrm{H} 12 \mathrm{~B}$ & 0.5756 & 0.0545 & 0.4510 & $0.026^{*}$ \\
\hline C13 & $0.2816(8)$ & $0.3188(5)$ & $0.2248(4)$ & $0.0256(16)$ \\
\hline $\mathrm{H} 13 \mathrm{~A}$ & 0.2366 & 0.3749 & 0.2049 & $0.031^{*}$ \\
\hline $\mathrm{H} 13 \mathrm{~B}$ & 0.2229 & 0.2723 & 0.1942 & $0.031^{*}$ \\
\hline C14 & $0.4536(8)$ & $0.3147(5)$ & $0.2333(4)$ & $0.0233(15)$ \\
\hline $\mathrm{H} 14 \mathrm{~A}$ & 0.4637 & 0.3001 & 0.1897 & $0.028^{*}$ \\
\hline H14B & 0.5042 & 0.3711 & 0.2488 & $0.028^{*}$ \\
\hline C15 & $0.3174(8)$ & $0.3891(5)$ & $0.3385(4)$ & $0.0245(15)$ \\
\hline $\mathrm{H} 15 \mathrm{~A}$ & 0.2546 & 0.3929 & 0.3675 & $0.029^{*}$ \\
\hline H15B & 0.2962 & 0.4410 & 0.3094 & $0.029^{*}$ \\
\hline C16 & $0.4943(8)$ & $0.3861(5)$ & $0.3842(4)$ & $0.0221(15)$ \\
\hline $\mathrm{H} 16 \mathrm{~A}$ & 0.5591 & 0.3960 & 0.3567 & $0.026^{*}$ \\
\hline $\mathrm{H} 16 \mathrm{~B}$ & 0.5192 & 0.4306 & 0.4200 & $0.026^{*}$ \\
\hline $\mathrm{Cl} 1$ & $0.3447(2)$ & $0.38910(11)$ & $0.51187(10)$ & $0.0271(4)$ \\
\hline $\mathrm{Cl} 2$ & $0.25233(19)$ & $0.16641(11)$ & $0.54815(8)$ & $0.0212(4)$ \\
\hline $\mathrm{Cl} 3$ & $0.7952(2)$ & $0.07337(12)$ & $0.29209(10)$ & $0.0280(4)$ \\
\hline $\mathrm{Cl} 4$ & $0.8692(2)$ & $0.30582(13)$ & $0.26128(9)$ & $0.0265(4)$ \\
\hline Co1 & $0.41717(10)$ & $0.21633(6)$ & $0.34264(4)$ & $0.0144(2)$ \\
\hline Co2 & $0.71356(9)$ & $0.23657(6)$ & $0.46548(4)$ & $0.0130(2)$ \\
\hline Co3 & $0.38507(10)$ & $0.24570(6)$ & $0.48920(5)$ & $0.0157(2)$ \\
\hline
\end{tabular}

Table 2 (continued)

\begin{tabular}{|c|c|c|c|c|}
\hline Atom & $x$ & $y$ & $z$ & $U_{\text {iso }} * / U_{\text {eq }}$ \\
\hline Co4 & $0.74714(10)$ & $0.21329(6)$ & $0.31869(5)$ & $0.0188(3)$ \\
\hline N1 & $0.2966(6)$ & $0.1196(4)$ & $0.2818(3)$ & $0.0201(12)$ \\
\hline $\mathrm{H} 1$ & 0.2160 & 0.1459 & 0.2472 & $0.024^{*}$ \\
\hline $\mathrm{N} 2$ & $0.8355(6)$ & $0.3309(4)$ & $0.5274(3)$ & $0.0187(12)$ \\
\hline $\mathrm{H} 2 \mathrm{C}$ & 0.9211 & 0.3032 & 0.5581 & $0.022^{*}$ \\
\hline N3 & $0.8600(7)$ & $0.1439(4)$ & $0.5130(3)$ & 0.020 \\
\hline H3 & 0.9603 & 0.1604 & 0.5185 & $0.024^{*}$ \\
\hline N4 & $0.2712(6)$ & $0.3082(4)$ & $0.2941(3)$ & $0.0188(12)$ \\
\hline $\mathrm{H} 4 \mathrm{C}$ & 0.1709 & 0.2922 & 0.2891 & $0.023^{*}$ \\
\hline 01 & $0.3081(5)$ & $0.1866(3)$ & $0.4005(2)$ & $0.0184(10)$ \\
\hline 02 & $0.2366(8)$ & $0.1053(7)$ & $0.1337(4)$ & $0.073(3)$ \\
\hline $\mathrm{H} 2$ & 0.2283 & 0.0808 & 0.0974 & $0.109^{*}$ \\
\hline 03 & $0.8241(5)$ & $0.2680(3)$ & $0.4087(2)$ & $0.0183(10)$ \\
\hline 04 & $0.9745(7)$ & $0.3354(4)$ & $0.6770(3)$ & $0.0391(14)$ \\
\hline $\mathrm{H} 4$ & 0.9165 & 0.3097 & 0.6932 & 0.059 * \\
\hline 05 & $0.6056(5)$ & $0.2062(3)$ & $0.5252(2)$ & $0.0171(10)$ \\
\hline 06 & $0.6103(5)$ & $0.1521(3)$ & $0.3945(2)$ & $0.0155(9)$ \\
\hline 07 & $0.5259(5)$ & $0.2478(3)$ & $0.2838(2)$ & $0.0173(10)$ \\
\hline 08 & $0.5214(5)$ & $0.3005(3)$ & $0.4138(2)$ & $0.0153(9)$ \\
\hline
\end{tabular}

coordination polymers are mainly dependent on the organic ligands and the metal ions [11-15]. So, through deliberate design and judicious choice of organic ligands and metal ions, such polynuclear metal complexes can be synthesized. Amino alcohols and their derivatives have been proved as powerful ligands in preparation of polynuclear metal complexes because their flexible coordination fashion and various coordination modes. The three heteroatoms can adjust their coordination number and orientation through rotation of $\mathrm{C}-\mathrm{N}, \mathrm{C}-\mathrm{O}$ and $\mathrm{C}-\mathrm{C}$ single bonds [16-19]. Herein, we reported a tetranuclear mixed valent cobalt complex.

The asymmetric unit of the title compound consists of two $\mathrm{Co}$ (II) ions (Co1, Co2), two Co(III) ions (Co3, Co4), four organic ligands, and four chlorido ligands. Both $\mathrm{Co} 1$ and $\mathrm{Co} 2$ exhibit a distorted octahedral coordination geometry, which is defined by two $\mathrm{N}$ atoms and four $\mathrm{O}$ atoms from three organic ligands, respectively. $\mathrm{Co} 3$ and $\mathrm{Co} 4$ are coordinated by two oxygen atoms from two organic ligands and two chlorido ligands, respectively, displaying the same distorted tetrahedral coordination geometries. In the crystal structure, there exist abundant hydrogen bonds, involving $\mathrm{N}-\mathrm{H} \cdots \mathrm{Cl}, \mathrm{O}-\mathrm{H} \cdots \mathrm{Cl}$ and $\mathrm{O}-\mathrm{H} \cdots \mathrm{O}$ hydrogen bonds forming a two-dimensional network.

Acknowledgements: This work was financial supported by Changsha Enviromental Protection College.

\section{References}

1. Bruker. APEX2, SAINT and SADABS. Brucker AXS Inc., Madison, Wisconsin, USA, 2009. 
2. Sheldrick, G. M.: Crystal structure refinement with SHELXL. Acta Crystallogr. C71 (2015) 3-8.

3. Makhankova, V. G.; Vassilyeva, O. Y.; Kokozay, V. N.; Skelton, B. W.; Reedijk, J.; Albada, G. A. V.; Soraced, L.; Gatteschid, D.: A unique heteropentanuclear $\mathrm{Cu}_{2}{ }_{2} \mathrm{Co}^{\mathrm{II}} \mathrm{Co}^{\mathrm{III}}{ }_{2}$ complex, synthesised from metallic $\mathrm{Cu}$ and $\mathrm{Co}$ acetate in the presence of triethanolamine. Magnetic properties and a strong $\mathrm{H}$-bond stabilised lattice. New J. Chem. 25 (2001) 685-689.

4. Bertaina, S.; Gambarelli, S.; Mitra, T.; Tsukerblat, B.; Müller, A.; Barbara, B.: Quantum oscillations in a molecular magnet. Nature 453 (2008) 203-206.

5. Stamp, P. C. E.; Gaita-Ariño, A.: Spin-based quantum computers made by chemistry: hows and whys. J. Mater. Chem. 19 (2009) 1718-1730.

6. Sanvito, S.: Molecular spintronics. Chem. Soc. Rev. 40 (2011) 3336-3355.

7. Goswami, S.; Mondal, A. K.; Konar, S.: Nanoscopic molecular magnets. Inorg. Chem. Front. 2 (2015) 687-712.

8. Bürgler, D. E.; He $\beta$, V.; Esat, T.; Fahrendorf, S.; Matthes, F.; Schneider, C. M.; Besson, C.; Monakhov, K. Y.; Kögerler, P.; Ghisolfi, A.; Braunstein, P.; Atodiresei, N.; Caciuc, V.; Blügel, S.: Spin-Hybrids: a single-molecule approach to spintronics. e-J. Surf. Sci. Nanotechnol. 14 (2016) 17-22.

9. Schmitz, S.; Monakhov, Y. K.; Leusen, J.; Izarova, N. V.; He $\beta$, V.; Kögerler, p.: $\mathrm{Co}^{\mathrm{II} / \mathrm{III}}$ horseshoe and $\mathrm{Ni}_{4}{ }_{4}$ lacunary cubane coordination clusters: the isobutyrate/ $N$-butyldiethanolamine reaction system. RSC Adv. 6 (2016) 100664-100669.

10. Sakiyama, H.; Chiba, H.; Tone, K.; Yamasaki, M.; Mikuriya, M.; Krzystek, J.; Ozarowski, A.: Magnetic Properties of a dinuclear nickel(II) complex with 2,6-bis[(2hydroxyethyl)methylaminomethyl]-4-methylphenolate. Inorg. Chem. 56 (2017) 138-146.

11. Yang, J.; Ma, J. F.; Liu, Y. Y.; Ma, J. C.; Batten, S. R.: A series of $\mathrm{Cu}(\mathrm{II})$ complexes based on different bis(imidazole) ligands and organic acids: formation of water clusters and fixation of atmospheric carbon dioxide. Cryst. Growth. Des. 8 (2008) 4383-4393.

12. Bu, X. H.; Tong, M. L.; Chang, H. C.; Kitagawa, S.; Batten, S. R.: A neutral 3D copper coordination polymer showing $1 D$ open channels and the first interpenetrating NbO-type network. Angew. Chem. Int. Ed. 43 (2004) 192-195.

13. Han, M. L.; Chang, X. H.; Feng, X.; Ma, L. F.; Wang, L. Y.: Temperature and $\mathrm{pH}$ driven self-assembly of $\mathrm{Zn}$ (II) coordination polymers: crystal structures, supramolecular isomerism, and photoluminescence. CrystEngComm 16 (2014) $1687-1695$.

14. Meng, X.; Song, S. Y.; Song, X. Z.; Zhu, M.; Zhao, S. N.; Wu, L. L.; Zhang, H. J.: A Eu/Tb-codoped coordination polymer luminescent thermometer. Inorg. Chem. Front. 1 (2014) 757-760.

15. Liu, H. Y.; Wu, H.; Ma, J. F.; Liu, Y. Y.; Yang, J.; Ma, J. C.: Inorganic-organic hybrid compounds based on octamolybdates and metal-organic fragments with flexible multidentate ligand: syntheses, structures and characterization. Dalton. Trans. 40 (2011) 602-613.

16. Chen, Y.; Liu, Q.; Deng, Y.; Zhu, H.; Chen, C.; Fan, H.; Liao, D.; Gao, E.: Vanadium, molybdenum, and sodium triethanolamine complexes derived from an assembly system containing tetrathiometalate and triethanolamine. Inorg. Chem. 40 (2001) 3725-3733.

17. Nesterov, D. S.; Makhankova, V. G.; Vassilyeva, O. Y.; Kokozay, V. N.; Kovbasyuk, L. A.; Skelto, B. W.; Jezierska, J.: Assembling novel heterotrimetallic $\mathrm{Cu} / \mathrm{Co} / \mathrm{Ni}$ and $\mathrm{Cu} / \mathrm{Co} / \mathrm{Cd}$ cores supported by diethanolamine ligand in one-pot reactions of zerovalent copper with metal salts. Inorg. Chem. 43 (2004) 7868-7876.

18. Nesterov, D. S.; Makhankova, V. G.; Kokozay, V. N.; Skelton, B. W.: Direct synthesis and crystal structures of new heteropolynuclear complexes containing aminoalcohol ligands: From heterobi- $(\mathrm{Co} / \mathrm{Zn})$ to heterotrimetallic $(\mathrm{Cu} / \mathrm{Co} / \mathrm{Zn})$ compounds. Inorg. Chim. Acta 358 (2005) 4519-4526.

19. Semenaka, V. V.; Nesterova, O. V.; Kokozay, V. N.; Zybatyuk, R. I.; Shishkin, O. V.; Boca, R.; Gómez-Garcí, C. J.; ClementeJuan, J. M.; Jezierska, J.: Structural and magnetic studies of tetranuclear heterometallic $\mathrm{M} / \mathrm{Cr}(\mathrm{M}=\mathrm{Co}, \mathrm{Mn})$ complexes self-assembled from zerovalent cobalt or manganese, Reineckes salt and diethanolamine. Polyhedron 29 (2010) 1326-1336. 